\title{
BIQ̊日-BYWORD
}

Proceedings of Anticancer Research

\section{Perioperative Management of Patients with Breast Cancer Complicated with Rheumatic Heart Disease}

\author{
Xing Lei, Liu Hong* \\ First Affiliated Hospital of Chongqing Medical University, Chongqing 400000, China
}

\section{ARTICLE INFO}

Article history:

Published online: 15th July, 2017

Key words:

Breast cancer

Rheumatic heart disease

Perioperative

Treatment

\footnotetext{
*Corresponding author
}

Liu Hong, First Affiliated Hospital of Chongqing Medical University, China. E-mall: 574662405@, qq.com

\section{ABSTRACT}

Objective: To investigate the perioperative treatment of patients with breast cancer complicated with rheumatic heart disease. Methods: The perioperative treatment of rheumatic heart disease in 2400 patients with breast cancer treated in our hospital from January 2007 to August 2011 were retrospectively analyzed. Results $\square$ Two of the 2400 patients with breast cancer had rheumatic heart disease, aged 71 and 55 years, respectively. One case with atrial fibrillation, and the other one case of mitral valve replacement valve, were treated with warfarin anticoagulant therapy. Two patients in the first 5 days before the withdrawal of warfarin, while low molecular weight heparin or fondaparinux sodium anticoagulant therapy, intraoperative careful hemostasis, as appropriate after the start of anticoagulant therapy. Patients within 24 hours after the incision area of ecchymosis, to adjust the anticoagulant drugs, to strengthen the drainage and hemostasis and other measures improved. Conclusion: Patients with breast cancer complicated with rheumatic heart disease are more complicated due to basic diseases, and their perioperative treatment is more complicated. Excessive anticoagulation or hemostatic treatment will lead to adverse reactions. Perioperative attention should be paid to monitor and adjust the patient INR value, intraoperative need to carefully hemostasis, as far as possible to stop bleeding, reduce coagulation, close observation of patients with local wound conditions, strengthen drainage, if necessary, add hemostatic, and more can be better The treatment effect. 


\section{Introduction}

Rheumatic heart disease referred to as rheumatic heart disease, is due to rheumatic fever activity, involving the heart valve caused by heart disease. Manifested as mitral, tricuspid, aortic valve with one or more valve stenosis and/or insufficiency. Often at the beginning of the disease is no obvious symptoms, the late manifestations of palpitation, shortness of breath, fatigue, cough, limb edema, cough pink foam sputum, until the heart failure ${ }^{[1]}$; some manifestations of valve thrombosis caused by arterial embolism such as pulmonary embolism and Cerebral infarction and life-threatening. Breast cancer with rheumatic heart disease patients due to the basic disease is more special, the perioperative treatment is also more complex, if not pay attention, it will lead to serious consequences. In this paper, we retrospectively analyzed 24000 patients with breast cancer from January 2007 to August 2011, and discussed the perioperative treatment of patients with breast cancer complicated with rheumatic heart disease for clinical reference.

\section{Materials and methods}

In our hospital from January 2007 to August 2011, more than 2400 cases of breast cancer were female, aged 2590 years (mean 49.6 years), according to the condition of neoadjuvant chemotherapy or postoperative adjuvant chemotherapy, such as CEF, TEC, NP program, etc .; surgical methods, including typical radical surgery, improved radical surgery, breast conserving surgery. Retrospective analysis of this group of more than 2400 cases of breast cancer in 2 patients with rheumatic heart disease in patients with perioperative treatment. Two patients had clear rheumatic heart disease before admission, aged 71 and 55 years, respectively, duration of 40 and 26 years, an average of 33 years. One case of atrial fibrillation, and the other one in 9 years before the mitral valve replacement surgery, both long-term oral warfarin anticoagulant therapy.

\section{Results}

\subsection{Preoperative conditions and treatment}

Case 1 patients due to "found left breast mass $2+$ years" admission, the core puncture diagnosis of breast cancer, $\mathrm{T}_{2} \mathrm{~N}_{0} \mathrm{M}_{0}$. Patients with rheumatic heart disease history of $40+$ years, no exchange valve treatment, previous oral warfarin $1.25 \mathrm{mg}$, qd, digoxin $0.25 \mathrm{mg}$, qd. At the time of admission, echocardiography showed severe mitral stenosis, aortic valve, mild tricuspid regurgitation, mild to moderate pulmonary hypertension, and left ventricular ejection fraction (EF) of 55\%. ECG icon: common type of atrial fibrillation. (PT) 23s (normal
$11 \mathrm{~s}-14.5 \mathrm{~s}$ ), international standardized ratio (INR) 2.04 (0.8-1.2), activated partial thromboplastin time (APTT) 53s (28s-44s). Platelet (PLT) $147 \times 109 /$ L. 5 days before surgery to disable warfarin, switch to low molecular weight heparin 4100IU support, $\mathrm{iH}$, qd, disabled the day before surgery. Preoperative blood clotting: PT 13.7s, INR 1.12, APTT 44.7s.

Case 2 patients due to "found right breast mass $2+$ month" admission, the core puncture diagnosis of breast cancer, T2N0M0. History of rheumatic heart disease 26 years, 9 years ago mitral valve valve replacement, previous oral warfarin $3.75 \mathrm{mg}$, qd. Atrialization, echocardiography: rheumatic heart disease, mitral valve replacement, mechanical valve function was abnormal, moderate aortic valve insufficiency, left atrial appendage abnormal echo, suggesting that the old wall thrombosis; EF 65 \%. ECG normal. Blood coagulation: PT 21.2s, INR 1.84, APTT 33.8s. PLT $72 \times 109$ / L. 5 days before surgery to disable warfarin, to be Andrews (fondi liver decanate) $2.5 \mathrm{mg}$, iH, qd, surgery day to disable. Preoperative blood clotting: PT 13.6s, INR 1.05, APTT 32.6s. PLT $67 \times 109 /$ L.

\subsection{Intraoperative conditions and treatment}

Two patients underwent radical mastectomy for breast cancer, intraoperative bleeding significantly, are to be carefully hemostasis. Case 1 routine use of electrocoagulation knife to stop bleeding. Case 2 on small blood vessels and active bleeding as far as possible the use of silk ligation, reduce the use of electric coagulation knife. Two patients with intraoperative wound hemostasis time than the average patient extended 20-30 minutes.

\subsection{Postoperative situation and treatment}

Case 1 on the first day after the emergence of the wound below the redness of ecchymosis, coagulation as shown: PT 13.8s, INR 1.13, APTT 36.4s. Blood red blood cells (RBC) decreased from $2.71 * 1012 / \mathrm{L}$ to $2.69 * 1012$ / $\mathrm{L}$, hemoglobin $(\mathrm{Hb})$ decreased from $111 \mathrm{~g} / \mathrm{L}$ to $80 \mathrm{~g} / \mathrm{L}$, so no anticoagulant therapy. RBC $3.19 * 1012 / \mathrm{L}$ and $\mathrm{Hb} 94 \mathrm{~g} / \mathrm{L}$ were examined after $800 \mathrm{ml}$ of erythrocyte suspension. Postoperative drainage is shown in Figure 1. Check the blood as normal, the second day after surgery plus blood clotting hemostasis. Followed by chest wall axillary drainage was significantly reduced in the first 8 days after removal of chest wall drainage tube. After 1 month of capecitabine (Xeloda) oral chemotherapy, and $1+$ months to continue to warfarin $1.25 \mathrm{mg}$ anticoagulant therapy, within 1 month after INR control in the 1.01-1.07. 


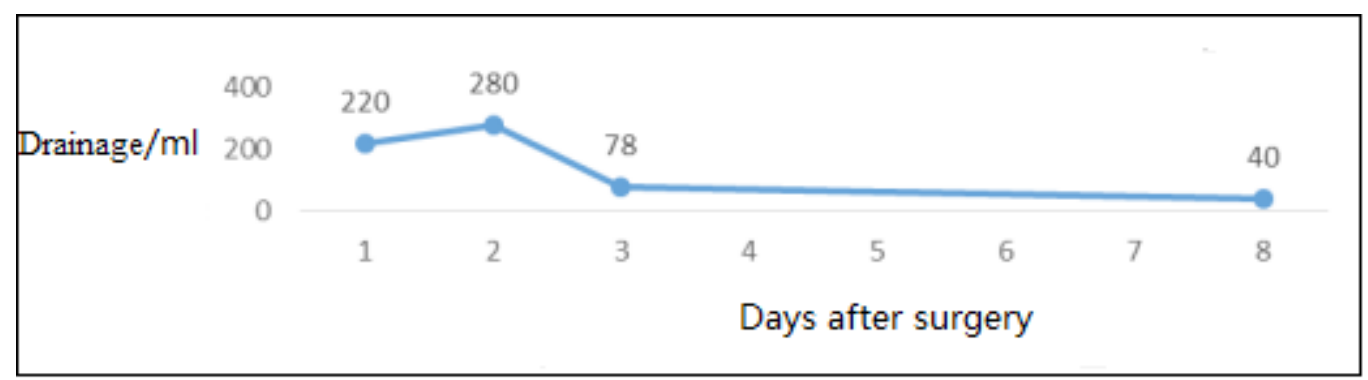

Figure 1 Case 1 postoperative wound drainage.

Case 2 patients, the surgeon believes that intraoperative hemostasis is good, and because of preoperative found left ear ear old wall thrombosis. 6 hours after surgery to be oral warfarin $2.5 \mathrm{mg}$, Andrews 1 subcutaneous anticoagulant therapy. The first day after surgery the patient appeared right chest wall near the axillary skin ecchymosis, enlargement, chest wall armor total bleed liquid $190 \mathrm{ml}$. Blood clotting: PT 13.6s, INR 1.05, APTT 32.2s. RBC from $3.6 * 1012$ / L before surgery to $3.03 * 1012$ / L, Hb from $109 \mathrm{~g} / \mathrm{L}$ down to $88 \mathrm{~g} / \mathrm{L}$, so the first day after surgery to disable warfarin, continue to Andrews treatment, within 4 days after INR Control at 1-1.09. The chest wall axillary drainage of clear liquid and gradually reduced, the first 5 days after the addition of warfarin $2.5 \mathrm{mg}$, qd, and in the first 7 days after surgery to $5 \mathrm{mg}$, qd, and in the first 8 days after removal of chest wall drainage tube. In addition to occasionally axillary drainage of blood, the right chest wall and axillary end of the ecchymosis did not increase. Since then, INR has risen to 1.93. Patients underwent chemotherapy with NP (cisplatin $60 \mathrm{mg}$, Gaino $80 \mathrm{mg}$ ) at 18 days postoperatively.

\section{Discussion}

Weibert RT et al. ${ }^{[2]}$ noted that excessive anticoagulation (INR $>5$ ) significantly increased the risk of bleeding when treated with warfarin anticoagulation in patients with rheumatic heart disease. However, a sudden decline in INR results in an increased risk of thrombosis in INR below treatment levels. Thus combined with rheumatic heart disease in patients with breast cancer perioperative treatment than the general breast cancer patients complicated. There is a cause of surgery or systemic bleeding or valve thrombosis and vascular thrombosis risk of life-threatening. At home and abroad on the merger of rheumatic heart disease in patients with breast cancer treatment of the literature reported less. Treatment of patients with rheumatic heart disease in patients with breast cancer perioperative treatment of anticoagulant hemostasis are discussed below.

\subsection{Preoperative preparation}

The American Valvular Heart Disease Treatment Guidelines indicate that patients with mitral stenosis and atrial fibrillation (paroxysmal, persistent, permanent), or mitral stenosis with previous embolization events, or mitral stenosis with left Patients with thrombosis are suitable for anticoagulant therapy (vitamin K antagonist VKA or heparin). Artificial mechanical valve replacement in patients with no risk of thromboembolism, recommended VKA anticoagulation therapy and make INR reach 2.5. Kearon $\mathrm{C}$ et al. ${ }^{[5]}$ considered that for most patients treated with warfarin, warfarin was discontinued 4 to 5 days prior to surgery or other invasive procedures to reduce preoperative INR to normal ), Patients with preoperative 2 to 3 days there is the risk of thrombosis. In patients with high risk of thrombosis in the 2 days before surgery to disable warfarin while oral administration of vitamin $\mathrm{K} 12.5 \mathrm{mg}$ $\mathrm{qd}$, INR can still be reduced to normal before surgery, but patients without protection time $<2$ days. At the same time preoperative use of low molecular weight heparin can also reduce the unprotected time, anticoagulant therapy can be reopened at the appropriate time after surgery. In this study, two patients, respectively, due to severe mitral stenosis and atrial fibrillation and mitral valve replacement surgery, were required warfarin anticoagulant therapy, INR was controlled at 2.04 and 1.84 or so, 5 days before surgery disabled, INR is controlled at 1.09 and 1.05, respectively, to meet the requirements of the guide. After the withdrawal of warfarin, patients were given low molecular weight heparin or Andrew anticoagulant therapy, and the day of surgery to disable low molecular weight heparin or Andrews, no thrombosis and other complications.

\subsection{Intraoperative treatment}

In this study, two patients before surgery, although INR control in the normal range, but the intraoperative wound is still bleeding significantly, hemostasis time than the conventional extension of 20-30 minutes. For such patients should be used to stop bleeding, reduce the application of an electro-coagulation knife. Nevertheless, the patient still bleeding after surgery, bleeding more. Breast cancer im- 
proved radical surgery incision a wide range of wound, bleeding more. The use of an electric knife to effectively improve the patient's intraoperative and postoperative wound bleeding. But for patients with rheumatic heart disease, especially preoperative oral warfarin in patients should not routinely use electrocoagulation knife to stop bleeding, small blood vessels and active bleeding and bleeding significantly as far as possible the use of silk ligation, so that hemostasis carefully, and should not pursue the speed of surgery to minimize the postoperative wound a lot of bleeding, in which bleeding and hematoma occurred.

\subsection{Postoperative treatment}

The American Heart Valve Disease Treatment Guidelines also point out that ${ }^{[3]}$, due to noncardiac surgery, invasive surgery or dental surgery need to interrupt the treatment of warfarin, if the risk of thrombosis in patients with low $24 \mathrm{~h}$ after the start of use. 2 If the patient has thrombotic risk factors (refers to the mitral or aortic valve mechanical valve replacement surgery), should be stable after the postoperative bleeding as soon as possible after the resumption of use until the INR returned to warfarin treatment level.

In this study, two patients with postoperative INR in the normal range, were the first day after surgery on the wound around the hematoma, ecchymosis, and postoperative chest wall axillary drainage of bloody fluid was significantly more than the average patient, chest wall drainage tube placement time is longer in compared with general patients. Two patients were followed up for hemoglobin which was decreased, indicating that such patient even if the risk of normal bleeding INR higher than the average patient. Which cases of patients with postoperative blood transfusion $800 \mathrm{ml}, 1+$ months without anticoagulant therapy, and the second day after the addition of blood coagulation enzyme treatment did not appear thrombosis and other adverse reactions, indicating that patients with postoperative bleeding significantly the time of anticoagulation therapy and the proper use of hemostatic agents that do not affect the amount of prothrombin in the blood are safe. Another case of patients with mitral valve replace- ment surgery, due to preoperative echocardiography left atrial appendage of the old wall thrombosis, and consider the effect of hemostasis surgery, so after 6 hours to restore the use of warfarin and Andrews treatment, Followed by local bleeding in patients with obvious, so the first day after surgery to disable warfarin and on the fifth day after surgery began to gradually restore warfarin treatment, no bleeding and thrombosis and other complications. Therefore, the recovery time of warfarin can be adjusted according to the patient's condition and local conditions of the wound, the use of low molecular weight heparin or sulton disulfide anticoagulation therapy when using warfarin.

\section{References}

[1] Xu Yuanxun. Rheumatic Heart Disease Heart Failure in 20 Cases of Clinical Efficacy Analysis. China Practical Medicine. 2011. 6 (22): 68-69.

[2] Weibert RT, Le DT, Kayser SR, Rapaport SI. Correction of Abnormal Anticoagulation with Low-Dose Oral Vitamin K1. Ann Intern Med. 1997. 126 (12): 959-62.

[3] Bonow RO, Carabello BA, Chatterjee K, et al. 2008 focused update incorporated into the ACC / AHA 2006 guidelines for the management of patients with valvular heart disease: a report of the American College of Cardiology / American Heart Association Task Force On Practice Guidelines (Writing Committee to revise the 1998 guidelines for the management of patients with valvular heart disease.) Endorsed by the Society of Cardiovascular Anesthesiologists, Society for Cardiovascular Angiography and Interventions, and Society of Thoracic Surgeons. J Am Coll Cardiol. 52 (13): e1-142.

[4] Nishimura RA, Otto CM, Bonow RO, et al. 2014 AHA / ACC guideline for the management of patients with valvular heart disease: a report of the American College of Cardiology / American Heart Association Task Force on Practice Guidelines. Coll Cardiol. 2014. 63 (22): e57-185.

[5] Kearon C, Hirsh J. Management of Anticoagulation before and after Elective Surgery. N Engl J Med. 1997. 336 (21): 1506-11. 